\title{
Uso do fluconazol endovenoso no tratamento da endoftalmite fúng’ica endógena: relato de caso
}

\author{
Intra venous fluconazole use in the treatmentoffungic endogenous endophthalmitis: \\ case report
}

Patrícia de Freitas Dotto ${ }^{1}$ Luciana Duarte Rodrigues ${ }^{2}$

Maria de Lourdes Brandão Fernandes ${ }^{3}$

Bettina Barbosa Duque Figueira ${ }^{4}$

Walter Yukihiko Takahashi $^{5}$

José Carlos Eudes Carani ${ }^{6}$

Pedro Durães Serracarbassa ${ }^{7}$
${ }^{1}$ Médica Pós-Graduanda em Oftalmologia pela Faculdade de Medicina da Universidade de São Paulo (USP). São Paulo (SP).

${ }^{2}$ Médica Pós-Graduanda em Oftalmologia pela Faculdade de Medicina da USP. São Paulo (SP).

${ }^{3}$ Médica do Serviço de Neonatologia do Hospital Municipal Carmito Caricchio.

${ }^{4}$ Médica do Serviço de Neonatologia do Hospital Municipal Carmito Caricchio.

5 Médico Assistente Doutor da Clínica Oftalmológica da Faculdade de Medicina da USP. São Paulo (SP)

${ }^{6}$ Médico Assistente Doutor da Clínica Oftalmológica da Faculdade de Medicina da USP. São Paulo (SP).

Doutor em Medicina pela Faculdade de Medicina da USP. São Paulo (SP).

Endereço para correspondência: Pedro D. Serracarbassa - Av. Brigadeiro Faria Lima 1903, cj. 43

São Paulo (SP) CEP 01472-001

E-mail: serracar@brfree.com.br

Recebido para publicação em 17.08.2004

Versão revisada recebida em 13.01.2005

Aprovação em 23.03.2005

Nota Editorial: Após concluída a análise do artigo sob sigilo editorial e com a anuência do Dr. Eduardo Cunha de Souza sobre a divulgação de seu nome como revisor, agradecemos sua participação neste processo.

\section{RESUMO}

Os autores descrevem um caso de endoftalmite endógena por Candida albicans, em recém-nascido prematuro, refratária ao tratamento com anfotericina B endovenosa e que apresentou resolução com o uso do fluconazol endovenoso. Ressaltam ainda os aspectos clínicos da endoftalmite endógena por Candida albicans por meio de revisão da literatura.

Descritores: Candidíase; Endoftalmite/quimioterapia; Infecções oculares fúngicas/quimioterapia; Prematuro; Candida albicans/isolamento \& purificação; Fluconazol/uso terapêutico

\section{INTRODUÇÃO}

A Candida albicans é o agente etiológico mais comum relacionado a endoftalmite fúngica endógena ${ }^{(1-4)}$, demonstrando-se altamente patogênica quando comparada a outras espécies ${ }^{(5)}$ na evolução desta doença. Nas últimas décadas, o aumento da incidência de endoftalmite fúngica endóge$\mathrm{na}^{(6)}$ sofreu influência de vários fatores, como a difusão da antibioticoterapia $^{(7-8)}$, a cateterização intravenosa ${ }^{(9-12)}$, o emprego da nutrição parenteral prolongada $^{(13)}$, a imunossupressão causada por doença sistêmica ou induzida por terapia imunossupressiva ${ }^{(4,14-16)}$, o uso de drogas ilícitas ${ }^{(17)}$ e a prematuridade ao nascimento ${ }^{(18-24)}$.

Os fungos têm emergido como importantes patógenos nas unidades de terapia intensiva neonatal, constituindo $0,9 \%$ do total de admissões, atingindo em especial os recém-nascidos prematuros de muito baixo peso, com incidência de infecção sistêmica de 2 a $5 \%$ (25).

$\mathrm{O}$ acometimento ocular na infecção fúngica sistêmica tem sido relatado em freqüências que variam de $9^{(26)}$ a $45 \%{ }^{(27)}$.

As características oftalmológicas associadas ao quadro clínico do paciente e a positividade da cultura extra-ocular para Candida sp são consideradas diagnósticas de endoftalmite fúngica. No entanto, nos recém-nascidos prematuros de muito baixo peso, estas culturas costumam ser negativas e o diagnóstico é usualmente baseado no aspecto fundoscópico da lesão ${ }^{(28)}$.

Atualmente novos métodos diagnósticos e opções utilizadas no tratamento das infecções fúngicas intra-oculares ${ }^{(29-31)}$ surgem como alternativas ao uso da anfotericina B.

\section{RELATO DE CASO}

Paciente do sexo masculino, branco, prematuro, referido ao Serviço de Oftalmologia do HC-USP aos 42 dias de vida para avaliação oftalmológica devido à infecção fúngica sistêmica, no $28^{0}$ dia de tratamento com anfoteri- 
cina B. A idade gestacional conceptual foi 36 semanas e o peso ao nascimento $1.730 \mathrm{~g}$, sendo pequeno para idade gestacional, com apgar:5-8, desconforto respiratório precoce, sepsis neonatal precoce e tardia, anemia, plaquetopenia, meningite, candidíase sistêmica e abscessos em couro cabeludo e no membro superior direito. Permaneceu sob ventilação mecânica por 33 dias, nutrição parenteral prolongada por 27 dias e flebotomia por 16 dias. Recebeu antibioticoterapia sistêmica (penicilina/amicacina; vancomicina/imipenem) nos primeiros trinta dias de vida. A anfotericina B foi associada no $14^{0}$ dia de vida (dose acumulada de $26,25 \mathrm{mg} / \mathrm{kg}$ ), após isolamento de Candida albicans na hemocultura e no produto de drenagem dos abscessos cutâneos. As culturas de ponta de cateter da flebotomia, da secreção contida no tubo de ventilação mecânica e da sonda nasogástrica foram negativas.

A avaliação oftalmológica foi realizada no $42^{\circ}$ dia de vida. No momento da avaliação, o paciente apresentava estado geral regular, mas os sinais sistêmicos demonstravam melhora clínica progressiva desde a instituição da anfotericina B. No exame ocular externo, observou-se hiperemia discreta em AO, com nítido aumento da Po do OD à digitopressão e aumento dos diâmetros corneano e axial. À biomicroscopia, o olho direito apresentava hiperemia conjuntival $2-3+/ 4+$, com injeção ciliar, aumento do diâmetro corneano e edema estromal 2+/ $4+$, flare $2+/ 4+$, seclusão pupilar e opacificação total do cristalino; o olho esquerdo apresentava discreta injeção ciliar, córnea lisa, branca e transparente, ausência de reação de câmara anterior, pupila regular e cristalino transparente. A fundoscopia do olho direito foi impossibilitada pela opacidade de meios e a câmara posterior foi avaliada através da ultrasonografia. À oftalmoscopia binocular indireta do olho esquerdo observou-se vítreíte 2-3+/4+ e lesão esbranquiçada no pólo posterior, nasal à papila e anteriorizada ao plano retiniano. A ultra-sonografia do olho direito foi compatível com aumento do comprimento axial, turvação vítrea em pólo posterior e aumento da escavação e do olho esquerdo, com presença de debris inflamatórios densos no pólo posterior e discreto espessamento da coróide. Foi instituído fluconazol na dosagem de $7 \mathrm{mg} / \mathrm{kg} /$ dia e suspensa a anfotericina B. O paciente foi submetido à reavaliação oftalmológica semanal, incluindo a ultra-sonografia em $\mathrm{AO}$ (Figuras 1 e 2). No $7^{\circ}$ dia de tratamento com fluconazol, observou-se diminuição da turvação vítrea e floculação no vítreo, sobre a retina, no pólo posterior do olho esquerdo. No $15^{\circ}$ dia de tratamento com fluconazol, o olho direito apresentava-se calmo, com regressão do edema corneano, ausência de hiperemia conjuntival e câmara anterior sem reação. A ultra-sonografia apresentava vítreo anecóico e a escavação permanecia aumentada. A oftalmoscopia do olho esquerdo era compatível com a normalidade, com desaparecimento da lesão.

\section{DISCUSSÃO}

A C. albicans pode ser adquirida tanto verticalmente, por via ascendente ou transvaginal, como horizontalmente, estando presente em $64 \%$ dos recém-nascidos prematuros de muito baixo

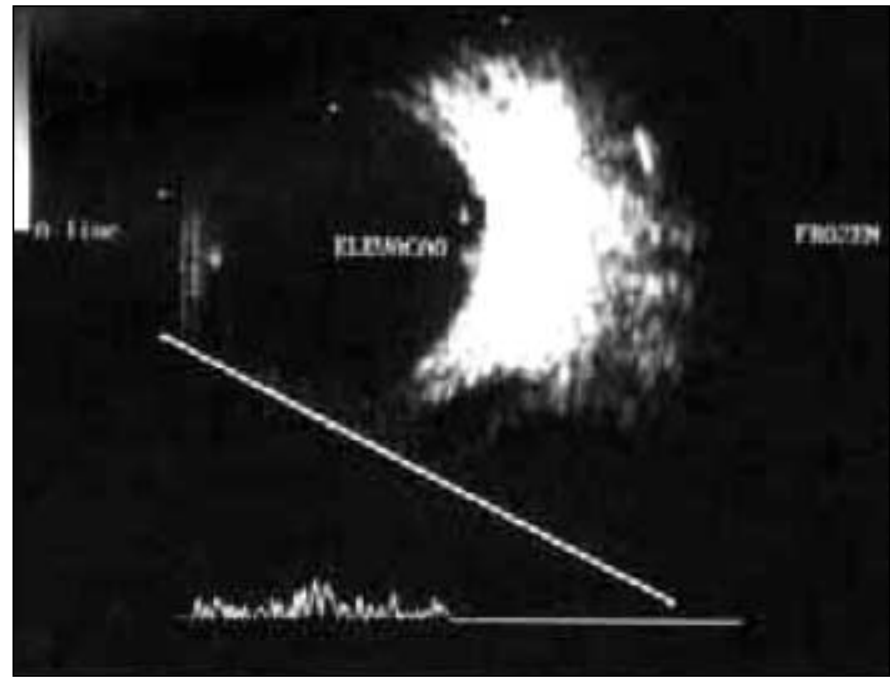

Figura 1 - Ecografia do olho direito. Aumento do comprimento axial, turvação vítrea no pólo posterior e aumento da escavação

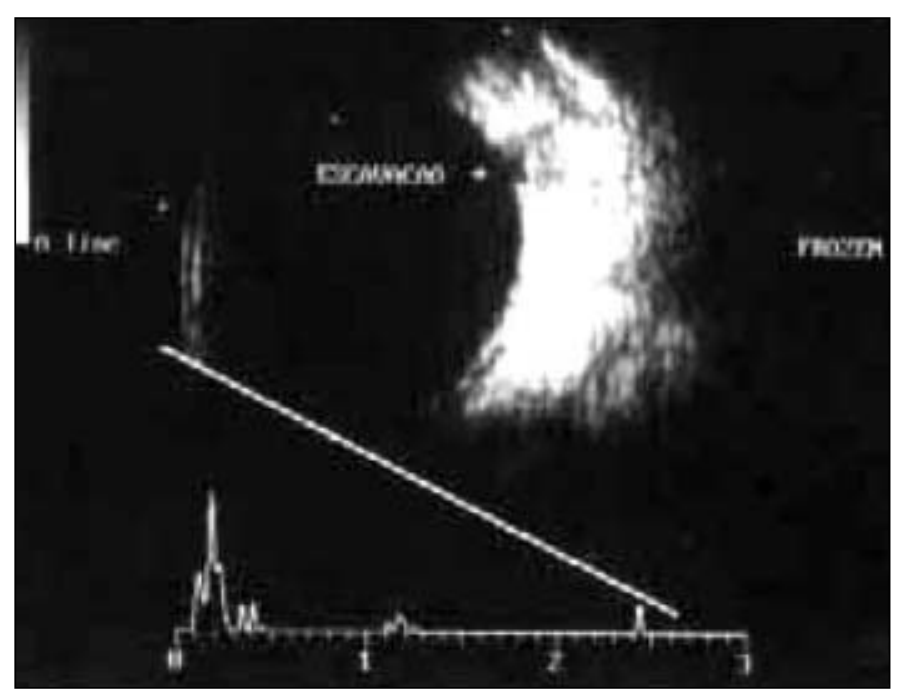

Figura 2 - Ecografia do olho esquerdo. Pontos hiperecogênicos móveis, de baixa refletividade sobre o pólo posterior (figura 2)

peso após a $4^{\underline{a}}$ semana de vida. Apenas $8 \%$ chegam a apresentar sepse fúngica e a maioria permanece assintomática ${ }^{(32)}$.

Por ser um habitante normal do trato gastrointestinal e regiões mucocutâneas, o crescimento da C. albicans é normalmente inibido pelas bactérias presentes no intestino ${ }^{(3)}$. Nas patologias onde a antibioticoterapia sistêmica prolongada é requerida, como no presente caso, há alteração da flora intestinal viabilizando a proliferação fúngica e sua disseminação por via hematogênica ${ }^{(33)}$. Os sinais sistêmicos associados a infecções fúngicas disseminadas em prematuros de muito baixo peso incluem temperatura corporal instável, hiperglicemia, dificuldade respiratória, distensão abdominal e hipotensão ${ }^{(33)}$. Freqüentemente, estas crianças são tratadas com nutrição parenteral, uso de cateteres e antibióticos de largo espectro. A prematuridade de nascimento do paciente e a exposição a 
vários fatores de risco como cateterização venosa, sondagem gástrica e ventilação mecânica propiciaram um ambiente favorável ao desenvolvimento da infecção fúngica ${ }^{(1)}$.

$\mathrm{O}$ acometimento ocular foi denotado por injeção conjuntival, injeção ciliar, precipitados ceráticos, células inflamatórias na câmara anterior e membrana pupilar. Estes sinais e outros, como membrana ciclítica, edema do nervo óptico e perivasculite podem fazer parte do quadro clínico da endoftalmite fúngica endógena ${ }^{(34)}$. O sinal oftalmológico característico é a presença de lesão branca, elevada, de superfície lisa e pouco delimitada, envolvendo a coróide e retina, localizada no pólo posterior, podendo ser única ou múltipla. Muitas vezes, essas lesões são circundadas por hemorragias intra-retinianas, levando ao aspecto das "manchas de Roth", e associadas a opacidades vítreas branco-amareladas, conectadas por traves, semelhante a "colar de pérolas"(22). Neste paciente só foram observadas opacidades vítreas floculares, não sendo possível quantificar o grau de comprometimento retiniano. As lesões da retina e coróide podem dar lugar a cicatrizes fibrosas com possível formação de membranas neovasculares, membrana epirretiniana, tração vítreo-retiniana e descolamento de retina $^{(34)}$. Raramente há opacificação do cristalino $^{(20)}$, como observado neste caso. O diagnóstico presuntivo de endoftalmite endógena no presente caso foi estabelecido a partir do quadro clínico ocular e da positividade para C. albicans, tanto da hemocultura quanto da cultura do produto de drenagem dos abscessos. A ultra-sonografia ocular foi útil na detecção de envolvimento do segmento posterior e também na monitorização da evolução do processo infeccioso, principalmente do olho direito, onde a opacificação total do cristalino impossibilitou a oftalmoscopia. São descritos ecos de baixa refletividade e alta mobilidade no vítreo, grandes vacúolos intravítreos e espessamento da hialóide posterior na maioria dos casos de endoftalmite fúngica ${ }^{(35)}$.

Apesar dos vários fármacos disponíveis no mercado atualmente, a anfotericina $\mathrm{B}$ ainda é o antifúngico de eleição ${ }^{(36)}$. A droga está disponível apenas para administração por via intravenosa. A disfunção renal é o mais importante efeito tóxico e costuma ser reversível quando cessada a terapia ${ }^{(37)}$. O tratamento sistêmico, em doses de $0,25 \mathrm{mg} / \mathrm{kg}$ a $1,5 \mathrm{mg} / \mathrm{kg}$ ao dia, é geralmente indicado nos casos de candidíase associada à coriorretinite sem opacidades vítreas. A vitrectomia via pars plana e a injeção intravítrea de 5 a $10 \mathrm{mg}$ de anfotericina B são as opções terapêuticas nos casos de opacidades vítreas moderadas ou severas. Nos casos de opacidades vítreas leves, sem associação com candidemia, considera-se somente o tratamento com injeções intravítreas da droga ${ }^{(38)}$. Especula-se que níveis subterapêuticos intravítreos, alcançados quando a anfotericina B é administrada por via endovenosa, sejam os responsáveis pelo fracasso terapêutico nos casos de infecções intra-oculares persistentes com envolvimento vítreo ${ }^{(38-39)}$. Entretanto, o quadro clínico sistêmico e o risco anestésico do paciente impossibilitaram a realização da injeção intravítrea ou vitrectomia. Dentre as opções terapêuticas ${ }^{(28)}$, o fluconazol ${ }^{(40)}$ apresenta-se altamente eficaz contra as espécies de Candida e de outros fungos, podendo ser administrado por via oral, na dosagem de 200 a $400 \mathrm{mg}$ ao dia, ou por via intravenosa.

No entanto, a penetração intravítrea do fluconazol administrado por via sistêmica é controversa na literatura ${ }^{(41)}$. Estudos mostraram que a monoterapia com fluconazol sistêmico foi eficaz no tratamento da endoftalmite endógena fúngica, com ou sem vitrectomia concomitante, inclusive com total desaparecimento das lesões ${ }^{(6,41-44)}$. Sendo assim, optou-se por esta modalidade terapêutica. Na falência terapêutica da anfotericina $\mathrm{B}$, a eficácia do fluconazol foi atribuída a um sinergismo entre as duas drogas ${ }^{(2)}$. Entretanto, modelos experimentais de endoftalmite por Candida em coelhos sugerem baixa eficácia do fluconazol sistêmico nos casos de endoftalmite decorrentes de candidíase disseminada, ocorrendo falência terapêutica entre $25-37,5 \%{ }^{(45-47)}$.

Ressalta-se aqui a importância da avaliação oftalmológica dos recém-nascidos com sepse fúngica, especialmente quando há acometimento meníngeo, pelo risco de complicações oculares potencialmente debilitantes que requerem intervenção precoce e porque a piora do quadro clínico ocular pode ocorrer a despeito da melhora sistêmica do paciente.

Mesmo sendo a anfotericina $\mathrm{B}$, atualmente, o antifúngico mais eficaz disponível, casos de resistência à droga são descritos. O uso de fluconazol endovenoso mostrou-se eficaz no tratamento da endoftalmite fúngica endógena no presente caso, sem a necessidade de vitrectomia e/ou injeção intravítrea. Estudos futuros são necessários no intuito de avaliar a eficácia desta modalidade terapêutica.

\section{ABSTRACT}

The authors describe a case of endogenous Candida albicans endophthalmitis in one extremely low birth-weight newborn refractory to endovenous amphotericin B treatment that presented resolution with the use of endovenous fluconazole. Clinical aspects of endogenous Candida albicans endophthalmitis are also pointed out by a review of the literature.

Keywords: Candidiasis; Endophthalmitis/drug therapy; Eye infections, fungal/drug therapy; Infant, premature; Candida albicans/isolation \& purification; Fluconazole/therapeutic use

\section{REFERÊNCIAS}

1. Clinch TE, Duker JS, Eagle RC Jr, Calhoun JH, Augsburger JJ, Fischer DH. Infantile endogenous Candida endophthalmitis presenting as cataract. Surv Ophthalmol. 1989;34(2):107-12.

2. Essman TF, Flynn HW Jr, Smiddy WE, Brod RD, Murray TG, Davis JL, et al. Treatment outcomes in a 10-year study of endogenous fungal endophthalmitis. Ophthalmic Surg Lasers. 1997;28(3):185-94.

3. Neves RA, Rigueiro MP, Bordon AF, Burnier M Jr. Endoftalmite micótica endógena: aspectos clínicos e histopatológicos de 3 casos. Arq Bras Oftalmol. 1990;53(1):20-2

4. Heinemann MH, Bloom AF, Horowitz J. Candida albicans endophthalmitis in a patient with AIDS: Case report. Arch. Ophthalmol. 1987;105(9):1172-3.

5. Fujita NK, Henderson DK, Hokey LJ, Guze LB, Edwards JR Jr. Comparative 
ocular pathogenicity of Cryptococcus neoformans, Candida glabrata, and Aspergillus fumigatus in the rabbit. Invest. Ophthalmol. Vis. Sci. 1982;22(3): 410-4.

6. Samiy N, D'amico DJ. Endogenous fungal endophthalmitis. Int Ophthalmol Clin. 1996;36(3):147-62.

7. Seelig MS. The role of antibiotics in the pathogenesis of Candida infections Am J Med. 1966;40(6):887-917.

8. Baley JE, Annable WL, Kliegman RM. Candida endophthalmitis in the premature infant. J Pediatr. 1981;98(3):458-61.

9. Klein JJ, Watanakunakorn C. Hospital-acquire fungemia: Its natural course and clinical significance. Am J Med. 1979;67(1):51-8.

10. Dellon AL, Stark WJ, Chretien PB. Spontaneous resolution of endogenous Candida endophthalmitis complicating intravenous hyperalimentation. Am J Ophthalmol. 1975;79(4):648-54.

11. Rose HD. Venous catheter-associated candidemia. Am J Med Sci. 1978;275(3): 265-9.

12. Henderson DK, Edwards JE Jr, Montgomerie JZ. Hematogenous Candida endophthalmitis in patients receiving parenteral hyperalimentation fluids. J Infect Dis. 1981;143(5):655-61.

13. McDonnell PJ, McDonnell JM, Brown RH, Green WR. Ocular involvement in patients with fungal infections. Ophthalmology. 1985;92(5):706-9.

14. Bodey GP. Infections in cancer patients. Cancer Treat Rev. 1975;2(2):89-128.

15. Wingard JR, Merz WG, Saral R. Candida tropicalis: a major pathogen in immunocompromised patients. Ann Intern Med. 1989;91(4):539-43.

16. Clift RA. Candidiasis in the transplant patient. Am J Med. 1984;77(Suppl 4D): 34-8.

17. Getnick RA, Rodrigues MM. Endogenous fungal endophthalmitis in a drug addict. Am J Ophthalmol. 1974;7(5):680-3.

18. Annable WL, Kachmer ML, DiMarco M, DeSantis D. Long-term follow-up of Candida endophthalmitis in the premature infant. J Pediatric Ophthalmol Strabismus. 1990:27(2):103-6.

19. Enzenauer RW, Calderwood S, Levin AV, Elder JE, Morin JD. Screening for fungal endophthalmitis in children at risk. Pediatrics. 1992;90(3):451-7.

20. Todd Johnston W, Cogen MS. Systemic candidiasis with cataract formation in a premature infant. JAAPOS. 2000;4(6):386-8.

21. Stern JH, Calvano C, Simon JW. Recurrent endogenous candidal endophthalmitis in a premature infant. JAAPOS. 2001;5(1):50-1.

22. Palmer EA. Endogenous Candida endophthalmitis in infants. Am J Ophthalmol. 1980;89(3):388-95.

23. Gago LC, Capone A Jr, Trese MT. Bilateral presumed endogenous Candida endophthalmitis and stage 3 retinopathy of prematurity. Am J Opthalmol. 2002;134(4):611-3.

24. Deconinck H, Libert J, Biarent D, Caspers-Velu LE. Endogenous mycotic endophthalmitis in an infant. Bull Soc Belge Ophthalmol. 1991;241:77-83.

25. Phillips G, Golledge C. Fungal infections in neonates. J Antimicrob Chemother. 1991;28(2):159-61.

26. Donahue SP, Greven CM, Zuravleff JJ, Eller AW, Nguyen MH, Peacock JE Jr, et al. Intraocular candidiasis in patients with candidemia. Clinical implications derived from a prospective multicenter study. Ophthalmology. 1994;101(7): 1302-9.

27. Chen JY. Neonatal candidiasis associated with meningitis and endophthalmitis. Acta Paediatr Jpn. 1994;36(3):261-5.
28. Serracarbassa PD. Estudo experimental da toxicidade retiniana e eficácia da injeção intravítrea de espartanamicina B no tratamento da endoftalmite por Candida albicans [tese].São Paulo: Faculdade de Medicina da Universidade de São Paulo; 2000.

29. Serracarbassa PD, Peyman GA, Liang C, Calixto N Jr, Nair MG. Toxicity and efficacy of intravitreal spartanamicin B in the treatment of Candida albicans endophthalmitis. Int Ophthalmol. 1998;22(1):53-8.

30. Shahsavari M, Peyman GA, Niesman MR. Retinal toxicity and in vitro efficacy study of cilofungin (LY121019). Ophthalmic Surg. 1990;21(10):726-8.

31. Dunlap WA, Karacorlu M, Peyman GA, Nair MG, Rahimy M, Pedroza L. Retinal toxicity of intravitreally injected faerifungin. Ophthalmic Surg. 1994; 25(5):303-3

32. Baley JE, Kliegman RM, Boxerbaum B, Fanaroff AA. Fungal colonization in the very low birth weight infant. Pediatrics. 1986;78(2):225-32

33. Edwards JE Jr, Foos RY, Montgomerie JZ, Guze LB. Ocular manifestations of Candida septicemia: review of seventy-six cases of hematogenous Candida endophthalmitis. Medicine (Baltimore). 1974;53(1):47-75.

34. Shah GK, Fischer D, Fineman MS, Sharma S, Maguire J. Subretinal neovascularization secondary to Candida endophthalmitis: long term follow-up after submacular surgery. Retina. 1999;19(1):81-2.

35. Dacey MP, Valencia M, Lee MB, Dugel PU, Ober RR, Green RL, et al. Echographic findings in infections endophthalmitis. Arch Ophthalmol. 1994; 112(10):1325-33.

36. Louria DB, Dineen P. Amphotericin B in the treatment of disseminated moniliasis. JAMA. 1960;174:273-9.

37. Butler WT. Pharmacology, toxicity and therapeutic usefulness of amphotericin B. JAMA 1966;195(5):371-5.

38. Peyman GA, Raichand M, Bennett TO. Management of endophthalmitis with pars plana vitrectomy. Br J Ophthalmol. 1980:64(7):472-5.

39. Griffin JR, Foos RY, Pettit TH. Relationship between Candida endophthalmitis, candidemia and disseminated candidiasis. In: $22^{\circ}$ Concilium Ophthalmologicum. Paris 1974. p.661.

40. Laatikainen L, Tuominen M, Von Dickhoff K. Treatment of endogenous fungal endophthalmitis with systemic fluconazole with or without vitrectomy. Am J Ophthalmol. 1992;113(2):205-7.

41. Smiddy WE. Treatment outcomes of endogenous fungal endophthalmitis. Curr Opin Ophthalmol. 1998;9(3):66-70.

42. Penk A, Pittrow L. [Status of fluconazole in the therapy of endogenous Candida endophthalmitis]. Mycoses. 1998;41(Suppl 2):41-4. Germany.

43. Mistlberger A, Graf B. [Endogenous candida endophthalmitis: a new therapy]. Klin Monatsbl Augenheilkd. 1991;199(6):446-9. Germany.

44. Luttrull JK, Wan WL, Kubak BM, Smith MD, Oster HA. Treatment of ocular fungal infections with oral fluconazole. Am J Ophthalmol. 1995; 119(4):477-81.

45. Nomura J, Ruskin J. Failure of therapy with fluconazole for candidal endophthalmitis. Clin Infect Dis. 1993;17(5):888-9.

46. Filler SG, Crislip MA, Mayer CL, Edwards JE Jr. Comparison of fluconazole and amphotericin B for treatment of disseminated candidiasis and endophthalmitis in rabbits. Antimicrob Agents Chemother. 1991;35(2):288-92.

47. Gupta SK, Dhingra N, Velpandian T, Jaiswa I. Efficacy of fluconazole and liposome entrapped fluconazole for C. albicans induced experimental mycotic endophthalmitis in rabbit eyes. Acta Ophthalmol Scand. 2000;78(4):448-50.

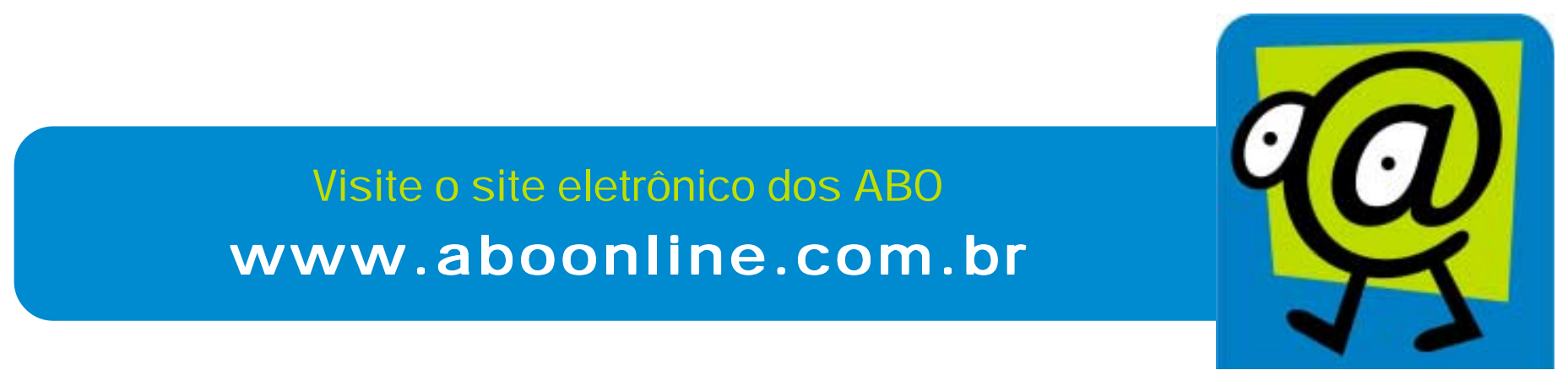

Arq Bras Oftalmol. 2005;68(4):543-6 